\title{
SOZIALISATION ALS SELBSTPROJEKT. EINE QUALITATIVE LÄNGSSCHNITTSTUDIE ZU AUSBILDUNG UND STUDIUM
}

Alexandra König

Universität Duisburg-Essen, Fakultät für Bildungswissenschaften

E-Mail: alexandra.koenig@uni-due.de

URL: https://www.uni-due.de/biwi/koenig/koenig.php

Zitationsvorschlag:

König, Alexandra (2020): Sozialisation als Selbstprojekt. Eine qualitative Längsschnittstudie zu Ausbildung und Studium. In: Gesellschaft - Individuum - Sozialisation (GISO). Zeitschrift für Sozialisationsforschung 1 (1). DOI: 10.26043/GISo.2020.1.2

Link zum Artikel:

https://doi.org/10.26043/GISo.2020.1.2

CC. $\$\left(\begin{array}{l}\text { BY } \\ \text { DC }\end{array}\right.$ 


\title{
SOZIALISATION ALS SELBSTPROJEKT. EINE QUALITATIVE LÄNGSSCHNITTSTUDIE ZU AUSBILDUNG UND STUDIUM
}

\author{
Alexandra König
}

Abstract: In dem Beitrag wird das Modell der "Sozialisation als Selbstprojekt“ vorgeschlagen, um Sozialisation gleichzeitig aus interaktionistischer und ungleichheitsanalytischer Perspektive zu erfassen. Der heuristische Wert des Modells wird am Beispiel einer qualitativen Längsschnittstudie vorgestellt, die sich der Ausbildung in kleinen Handwerksbetrieben sowie dem Lehramts- und Ingenieursstudium an Hochschulen zuwendet. Auf einer ersten Ebene wird die Strukturiertheit des jeweiligen Feldes untersucht. Hochschulen wie auch Betriebe werden als Felder im Sinne Bourdieus gefasst. Dieses Konzept erlaubt, das relationale Gefüge zu untersuchen und die Akteure in diesem zu verorten. Auf einer zweiten Ebene wird Sozialisation als interaktives Geschehen betrachtet. Im Fokus stehen dabei Strategien der Statusplatzierung aber grundsätzlicher auch der Teilhabe am Spiel. Auf einer dritten Ebene wird anschließend Sozialisation als Prozess der Selbstformung untersucht, im Sinne der Bearbeitung des Selbst als einer für sich und andere erkennbare Person. Im Dialog zwischen theoretischen Vorannahmen und empirischem Material werden die drei genannten Ebenen ausgearbeitet und im Modell der „Sozialisation als Selbstprojekt" zusammengeführt.

Keywords: Ungleichheit, Interaktionismus, Bourdieu, Ausbildung, Studium, Längsschnitt

\section{SOZIALISATION ALS VORBEREITUNG AUF GESELLSCHAFTLICHE TEILHABE}

Gemein ist jungen Erwachsenen, dass sie früher oder später die allgemeinbildende Schule verlassen und entscheiden müssen, wie es danach weitergehen soll. Mit der Aufnahme von Studium oder Ausbildung betreten sie dann sehr unterschiedliche Felder, in denen sie die nächsten Jahre - so zumeist der Plan - verbringen und sich, mehr oder weniger gezielt, auf ein bestimmtes berufliches Feld und einen beruflichen (und damit auch gesellschaftlichen) Status vorbereiten. Eine solche Vorbereitung kann als Sozialisation gefasst werden. Sozialisation „meint den Prozess, in dem die Individuen Schritt für Schritt die Fähigkeit, das Wissen, die Wertorientierungen und Motivationen erwerben, um an Gesellschaft teilzunehmen" (Bühler-Niederberger 2019: 136; vgl. auch Abels/König 2016: 1).

In Bezug auf die interessierende Statuspassage stellt sich die Frage, wie Sozialisation in Settings verläuft, in denen auf sehr unterschiedliche Berufe (und damit auch soziale Positionen) vorbereitet wird. Der vorliegende Beitrag basiert auf einer Studie, in der ausgewählte duale Ausbildungsgänge in Betrieben und Studiengänge an Hochschulen in den Blick genommen werden. Ziel ist es, aus einer sozialisationstheoretischen Perspektive Ungleichheiten der Statuspassage zu erfassen, aber auch zu untersuchen, wie Ungleichheiten in der Statuspassage produziert werden. Grundgedanke ist, dass zwar Bildungszertifikate „zentraler Mechanismus für die soziale Zuteilung von Lebenschancen" (Hillmert 2014: 82) sind, gleichwohl Ungleichheiten bereits durch das vorgelagerte Sozialisationsgeschehen in Ausbildung und Studium entstehen. Mit dem vorliegenden Beitrag ist nicht der Anspruch verbunden, die Unterschiede zwischen den Statuspassagen systematisch herauszuarbeiten das ist an anderer Stelle geschehen (König 2019). Vielmehr soll hier ein heuristisches Modell ${ }^{1}$ vorgestellt werden, mit dem das Sozialisationsgeschehen in unterschiedlichen Settings

\footnotetext{
${ }^{1}$ Die drei vorgestellten Ebenen des Modells haben die Analyse nicht strukturiert, sondern sind im Forschungsprozess als hilfreiche Heuristik entwickelt worden.
} 
untersucht werden kann. Gelenkt wird der Blick mit dieser Heuristik erstens auf das Ordnungsgefüge, in dem sich die jungen Erwachsenen während ihrer Ausbildung bzw. ihres Studiums befinden, zweitens auf die (interaktive) Ausgestaltung der Statuspassage und drittens die Weise, wie die jungen Erwachsenen sich und ihre Zukunft im Zuge der Statuspassage (neu) entwerfen.

Fokussiert werden in dem vorliegenden Beitrag zwei duale Ausbildungsberufe im Handwerk, nämlich das Maler-LackiererInnen- und Friseurlnnenhandwerk, sowie die universitäre Vorbereitung zum/zur Lehrerln und Ingenieurln. Skizziert wird zuerst der Forschungsstand zu nachschulischen Ausbildungswegen, insofern er für die eigene Untersuchung relevant ist (Kapitel 2). Daran schließt die Beschreibung des Forschungsprojekts an (Kapitel 3). Im Anschluss werden die zentralen Dimensionen des Modells der "Sozialisation als Selbstprojekt“ entfaltet (Kapitel 4), welche die Darstellung der Ergebnisse strukturiert (Kapitel 5). Die Arbeit schließt mit einem zusammenfassenden Fazit zum heuristischen Wert des vorgeschlagenen Modells.

\section{FORSCHUNGSSTAND: HOCHSCHULEN UND AUSBILDUNGSBETRIEBE ALS FELDER DER SOZIALISATION}

Schaut man sich die Forschungslage zu nachschulischen Bildungswegen an, so fällt eine gewisse (institutionell gestützte) Arbeitsteilung auf zwischen Forscherlnnen, die sich der dualen Ausbildung, und solchen, die sich dem Hochschulstudium zuwenden. Verbreitet sind in beiden Forschungsfeldern quantifizierende Studien aus ungleichheitstheoretischer Perspektive. Darin werden zahlreiche Belege aufgeführt, dass die Aufnahme von Studium bzw. Ausbildung (bzw. die Fächerwahl) wie auch der Abschluss bzw. Abbruch von der sozialen Herkunft abhängen (etwa: Bourdieu/Passeron 1971; Becker 2009; Isleib 2019; Reißig 2015: 198).

Weniger wissen wir darüber, was zwischen den Schwellen geschieht, wie das herkunftsspezi- fische Erbe in Bildungserfolg umgewandelt wird - oder grundlegender: wie Sozialisation in Ausbildung und Studium verläuft. ${ }^{2}$ Zum Studium ist die Forschungsbasis breiter, insbesondere zu BildungsaufsteigerInnen liegen aus habitusanaIytischer Perspektive einige Studien vor. Schmitt (2010) geht beispielsweise den „Habitus-Struktur-Konflikten“ nach. Darunter versteht er „Konflikte zwischen verinnerlichten kulturellen Mustern und solchen der Umgebung" (ebd.: 59), die er vor allem bei Studierenden aus Nicht-Akademikerhaushalten (ebd.: 267 f.) identifiziert. Reay, Crozier und Clayton sprechen in ähnlicher Weise von einer "out of field“-Erfahrung der „working-class students in the middle-class field of higher education" (2009: 1110). Lehmann (2009) kann am Beispiel von Studierenden der Arbeiterklasse an kanadischen Universitäten zeigen, wie die eigene soziale Herkunft nicht bloß als Nachteil gedeutet, sondern zu einem moralischen Vorteil umgedeutet wird. Diese an Bourdieu orientierten Arbeiten liefern wichtige Einblicke in die Relevanz der sozialen Herkunft. Entsprechend der theoretischen Voreinstellung geht es vor allem um die (mangelnde) Vorangepasstheit an ein vorstrukturiertes Feld.

Weniger Beachtung wird den interaktiven Anpassungs- und Aushandlungsprozessen von Studierenden geschenkt, die mit der Statuspassage alle ein unbekanntes Feld betreten. Für diese Fragerichtung sind solche Studien anregend, die in der Tradition der Chicago School of Sociology stehen. Exemplarisch sei die Studie "Boys in White" (Becker et al. 1961) genannt. Hier geht es um die Herausbildung einer "studentischen Kultur" bzw. "kollektiven Perspektive“ im Zuge des Studiums. Basis ist eine Ethnographie im Medizinstudiengang der Universität von Kansas. Nachgezeichnet wird, wie sich die Perspektive der Studierenden - im Sinne eines „coordinated set of ideas and actions a person uses in dealing with some problematic situation" (ebd.: 34) - im Laufe des Studiums verschiebt und Strategien an die wahrgenommenen Anforderungen des Studiums angepasst werden. Wenig erfahren wir über die ungleiche Ausstattung der „Boys in White" und ihre ungleichen Chancen, zu

\footnotetext{
${ }^{2}$ Statt die soziale Herkunft als Ursache für den Bildungserfolg zu setzen, schlägt Emmerich aus differenzierungstheoretischer Perspektive vor, "die Funktion von Organisationen als Operatoren gesellschaftlicher Ungleichheit“ (2016: 126) in Rechnung zu stellen.
} 
reüssieren. Der Wert der Studie liegt vor allem in den Erkenntnissen zum interaktiven Geschehen, der Modifizierung der Situationsdefinition und der Anpassung der Strategien.

Mit Blick auf Studien zum Studium ist ein dritter Forschungsstrang zu nennen, der in Anbetracht meiner sozialisationstheoretischen Perspektive nicht unerwähnt bleiben darf: Forschung zur Hochschulsozialisation (Friebertshäuser 2008: 621) und Fachkulturforschung. Exemplarisch genannt werden kann hier die Monographie von Engler (1993), in der das Zusammenspiel von Fachkultur, Geschlecht und sozialer Reproduktion untersucht wird. Während einige die konstatierten Zusammenhänge zwischen Fachkultur und Lebensstilen statistisch nur schwach bestätigt finden (Georg et al. 2009: 362 ff.), stellen andere die Annahme in Zweifel, dass Kulturmuster vom Fach abhängen (Niederbacher et al.: 2010). Angenommen wird vielmehr, dass das Studium an Dominanz im Leben von Studierenden verloren hat (Stichweh 2016). Welche Folgen dies für die Selbstentwürfe und Selbstformung (als StudentIn, als künftige/r Ingenieurln) hat, darüber ist wenig bekannt.

Studien zum Ausbildungsgeschehen verfolgen andere Erkenntnisinteressen: Fragen, wie die nach der Vorangepasstheit an ein bestimmtes Feld - etwa einer habituellen Nicht-Passung von Kindern aus Akademikerfamilien -, sind nicht Gegenstand dieser Untersuchungen. Ein Fokus liegt hingegen auf problematischen bzw. prekären Verläufen (zur Kritik daran: Klaus 2012), wobei interaktive Anpassungs- und Aushandlungsprozesse vor allem im Sinne von Bewältigungsstrategien (eng) gefasst werden (etwa: Kreher 2007). Vergleichbar mit der Fachkulturforschung sind Ansätze zur beruflichen Sozialisation und zu spezifischen Berufskulturen. Wegweisend sind hier Studien des Sonderforschungsbereichs (SFB) 186. Zentrale Fragestellung ist: „What kinds of aspirations and strategies do adolescents and young adults develop when faced with these different occupational contexts?" (Heinz et al. 1998: 93). Die entstandenen
Untersuchungen unterstreichen, dass die Selbstentwürfe junger Erwachsener vor dem Hintergrund der jeweiligen Berufskulturen kontextualisiert und in ihrem Wandel erfasst werden müssen.

Insgesamt bleibt festzuhalten, dass die Arbeitsteilung zwischen Hochschul- und Ausbildungsforschung dazu führt, dass je andere Fragen gestellt werden. Die vorliegende Studie will hingegen Studierende und Auszubildende unter einer gemeinsamen sozialisationstheoretischen Perspektive betrachten. Begründet wird dies damit, dass die Statuspassage (Glaser/Strauss 2010) die jungen Erwachsenen eint. Gleichwohl differenzieren sich die Wege der jungen Erwachsenen mit der Aufnahme von Studium oder Ausbildung zunehmend aus; sie betreten Felder, die ganz unterschiedliche Anforderungen, Zumutungen und Chancen bereithalten. Die Ungleichheiten der Statuspassage wie auch die Ungleichheiten, die diese produzieren, geraten unter der gemeinsamen Betrachtung in den Blick.

\section{DAS FORSCHUNGSPROJEKT: ZWISCHEN SELBSTPROJEKT UND LIMITIERTEN CHAN- CEN}

Basis der Argumentation ist eine abgeschlossene DFG-Studie. ${ }^{3}$ Kernkonzept der Studie ist das "Selbstprojekt". Gefasst wird hiermit die Art und Weise, wie junge Erwachsene die Statuspassage Ausbildung/Studium ausgestalten und zu sich in Bezug setzen. Mit dem Kernkonzept sind bereits die sozialtheoretischen Wurzeln der Studie benannt. Nach Mead (1973) ist das Selbst eine Instanz, die ständig geformt und dabei modifiziert wird, in Interaktion mit Anderen und in Auseinandersetzung mit gesellschaftlichen Erwartungen. Um das Selbstprojekt als (Sozialisations-)Prozess zu erfassen, folgt die Studie einem längsschnittlichen Design. Erhoben wurden Selbstprojekte über einen quantitativen und qualitativen Längsschnitt, mit insgesamt drei Erhebungszeitpunkten: Zu Beginn von Ausbildung und Studium, nach einem Jahr und wieder ein

\footnotetext{
${ }^{3}$ Das Projekt „Zwischen Selbstprojekt und limitierten Chancen - eine Längsschnittstudie der biographisch verdichteten Phase zwischen Schule und Berufsqualifikation" wurde 2008-2011 von der DFG finanziert (BU 1034/7-1). Bereits im Antrag, der von Bühler-Niederberger gestellt wurde, findet sich das Selbstprojekt als Konzept, welches im Forschungsverlauf empirisch fundiert bearbeitet wurde.
} 
Jahr später. Der letzte Erhebungszeitpunkt geht mit dem erwartbaren Abschluss der Ausbildung bzw. des BA-Studiengangs einher.

Für den vorliegenden Beitrag werden zwei Gruppen fokussiert: Auszubildende im Handwerk, genauer gesagt im Friseursalon und MalereiLackier-Betrieb, sowie Studierende in einem ingenieurswissenschaftlichen Studiengang bzw. im Lehramtsstudiengang (zur kontrastiven Anlage des Gesamtsamples: König 2019). Die Differenzen zwischen den Ausbildungsgängen bzw. zwischen den Studiengängen stehen im Folgenden nicht im Vordergrund. Die Zusammenfassung der beiden Handwerke wie auch der beiden Studiengänge lässt sich wie folgt begründen: Ausbildungen im Friseursalon sowie Malerei- und Lackier-Betrieb sind vor allem Wahlen von Hauptschülerlnnen - erstere sind weiblich dominiert, letztere männlich. Niedrig ist in beiden Fällen - im Vergleich zu anderen Handwerken - die Ausbildungsvergütung, wie auch der soziale Status von Gesellen und Gesellinnen (ISEI=29). Gleichwohl erscheinen diese Ausbildungen vielerorts als sichere Wahl, wie die häufige Bemühung des Ausspruchs „Handwerk verspricht goldenen Boden" indiziert. Lehramt und Ingenieurswissenschaften werden vor allem von jungen Erwachsenen gewählt, die nicht aus einem Akademikerlnnenhaushalt kommen - ersteres ist weiblich dominiert, letzteres männlich. Zum Zeitpunkt der ersten Befragung waren sowohl der Mangel an Lehrkräften wie auch an Ingenieurlnnen in aller Munde. Das heißt, beide Wahlen versprechen sichere Berufseinstiege. Der Berufsstatus ist ähnlich hoch (ISEI: ca. 70). Hauptziel der Gegenüberstellung der Auszubildenden und Studierenden ist im vorliegenden Beitrag nicht, systematisch deren Unterschiede herauszuarbeiten. Vielmehr wird an ihrem Beispiel eine Heuristik vorgestellt, die erlaubt, so unterschiedliche Sozialisationssettings wie Hochschulen und Betriebe zu analysieren und damit auch zu vergleichen.

Gestartet wurde die Erhebung mit einer quantitativen Befragung, aus der analytisch interessante Personen für die qualitativen Samples ausgewählt wurden. Dazu zählen beispielsweise objektiv unwahrscheinliche Personen - etwa eine Abiturientin im Malerei-Lackier-Betrieb wie auch Personen mit weniger überraschenden Merkmalen - etwa der Hauptschüler im MalereiLackier-Handwerk, dessen Bruder im gleichen Sektor arbeitet. Das qualitative Sample der Auszubildenden umfasst in der ersten Erhebung 27 junge Erwachsene, für die zweite Befragung konnten 23 Personen erneut gewonnen werden. Die Studierendengruppe setzt sich zuerst aus 16 Personen zusammen, in der zweiten Runde sind 10 Personen wiederholt befragt worden. Geführt wurden problemzentrierte Interviews (Witzel 2000) zur Ausgestaltung des Selbstprojekts. In der ersten Befragung $\left(t_{1}\right)$ geht es vor allem um den Prozess der Entscheidung für die Ausbildung bzw. das Studium sowie um das Einfinden in der neuen Welt; in der zweiten Befragung $\left(t_{2}\right)$ ist der weitere Ausbildungsverlauf Thema, und die jungen Erwachsenen, zumeist kurz vor Abschluss stehend, werden zu einer Bilanzierung und einem Zukunftsentwurf aufgefordert. ${ }^{4}$

\section{THEORETISCHE PERSPEKTIVE: DREI EBE- NEN DER SOZIALISATION ALS SELBSTPRO- JEKT}

Um Sozialisationsprozesse in den kleinen Ausbildungsbetrieben des Handwerks und in studierendenstarken Studiengängen an Hochschulen zu untersuchen und miteinander vergleichen zu können, wird das Modell der "Sozialisation als Selbstprojekt" vorgeschlagen. Das vorgeschlagene Modell weist eine deutliche Nähe zu dem Modell der "Sozialisation als generationales Ordnen" auf (Bühler-Niederberger/Türkyilmaz 2014). Allerdings steht in letzterem - das zeigt der Titel bereits an - das generationale Ordnen im Vordergrund. Die generationale Ordnung kann auch in den untersuchten Feldern von Ausbildung/Studium wesentliches Strukturierungsprinzip sein, allerdings wird - über das Feldkonzept - erst einmal offen gefragt, was die Position im Gefüge bestimmt.

\footnotetext{
${ }^{4}$ Die Forschung orientiert sich an der Grounded Theory, wie sie von Strauss (1991) vorgeschlagen wird, etwa in Hinblick auf die Kodierverfahren, die methodologische Positionierung und (in modifizierter Weise) das theoretical sampling (zum methodischen Vorgehen: König 2019).
} 
Mit dem Modell der „Sozialisation als Selbstprojekt" werden drei Ebenen analytisch voneinander getrennt:

Sozialisation innerhalb eines spezifisch strukturierten Feldes: Auf einer ersten Ebene steht die Strukturiertheit des Sozialisationssettings im Fokus. Hierfür werden der Ausbildungsbetrieb wie auch die Hochschule als Feld gefasst, d.h. als „Konfiguration von objektiven Relationen zwischen Positionen" (Bourdieu/Wacquant 1996: 127). Mit Bourdieu, der dieses Konzept generiert und empirisch fundiert hat, bestimmen sich die Positionen in einem Feld über das Kapital, das in dem jeweiligen Feld als wertvoll gilt. Das Geschehen innerhalb eines Feldes fasst Bourdieu als einen ständigen Kampf um die Bewahrung oder Verbesserung der eigenen Position im Feld (Bourdieu 2001a: 196). Grundvoraussetzung für die Teilnahme ist das Interesse an dem Spiel bzw. der Glaube an dessen Sinnhaftigkeit - die illusio (ebd.: 129). Gespielt wird nach feldspezifischen, nicht-kodifizierten Regeln. Einen Betrieb oder eine Hochschule als Feld zu fassen, mag auf den ersten Blick ungewöhnlich erscheinen, sofern man zuerst an die zentralen Feldanalysen von Bourdieu denkt, wie die zum literarischen oder wissenschaftlichen Feld. Diese Felder sind andere Gebilde als der Betrieb oder die Hochschule (bzw. der Studiengang in dieser). Allerdings findet man auch in Bourdieus Gesamtwerk Feldanalysen, die dem hiesigen Vorgehen ähneln. Erinnert sei an seine Studien zum Eigenheim (Bourdieu 2002), in der ein einzelnes Unternehmen als autonomes Feld gefasst wird. Was ein Feld ist, ist letztendlich eine empirische Frage (Bourdieu/Wacquant 1996). In der vorliegenden Studie dient das (adaptierte) Feldkonzept dazu, die Strukturiertheit des Settings und die Situiertheit des Handelns der jungen Erwachsenen in einem relationalen Gefüge zu erfassen.

Sozialisation als interaktives Geschehen (der Statusplatzierung): Wenn mit Bourdieu das Geschehen im Feld vor allem als Kampf um Positionen verstanden wird, kommen vor allem Strategien der Statusplatzierung in den Blick. Diese werden auch im vorliegenden Projekt untersucht, allerdings sollen aus einer stärker interaktionistischen Perspektive auch andere Teilnahmestrategien (etwa Kooperationen) erfasst werden. Relevanz erhalten in der Analyse die signifikanten und generalisierten Anderen sowie der Kommunikationsprozess in den jeweiligen Situationen bzw. in dem "game" (Mead 1973: 193 f.). Annahme ist, dass die Teilnahme am Spiel sowie die Fähigkeit zur Perspektivenübernahme dem Einzelnen ermöglichen, Hinweise zu suchen, "was er in einer spezifischen Situation zu tun hat" (ebd.: 317). Mit dieser zweiten Ebene wird also erfasst, um was es in den jeweiligen Spielen aus Sicht der jungen Erwachsenen geht, was für die Integration in das Spiel notwendig ist und welche Strategien nützlich sind. Um das Sozialisationsgeschehen zu verstehen, werden nicht nur die Feldteilnehmenden beachtet, sondern auch signifikante Andere, die am Spielfeldrand stehen und das Geschehen beeinflussen - sei es die Mutter, die zum Durchhalten der Ausbildung animiert, oder die Freundin, die sich trennt und damit die Fortsetzung des Studiums mühselig werden lässt. Grundlegend ist in Bezug auf das interaktive Handeln die Vorstellung, dass im Handeln an der (feldspezifischen) Ordnung gearbeitet wird - im Sinne einer Komplizenschaft, wie sie Bourdieu (2001b: 166) vorstellt, aber auch der Modifizierung, wie sie grundsätzlich mit dem „processual ordering“ nach Strauss (2014: 254 ff.) berücksichtigt ist.

Sozialisation als ein Prozess der Selbstformung: Auf einer dritten Ebene wird der Akteur selbst in den Fokus genommen, genauer gesagt: die Formung des Selbst. Es geht demnach nicht nur um die habituelle Passung, sondern auch um eine interaktive Anpassung an das Feld oder Abgrenzung von spezifischen Feldsegmenten bzw. dem Feld im Ganzen. Hierfür bietet sich der Begriff des Selbst als eine Instanz an, die in Auseinandersetzung mit signifikanten und generalisierten (hier: feldspezifischen) Anderen Kontur erhält. Nach Mead entsteht erst so ein Bewusstsein des Selbst: „Der Einzelne erfährt sich - nicht direkt, sondern nur indirekt - aus der besonderen Sicht anderer Mitglieder der gleichen gesellschaftlichen Gruppe oder aus der verallgemeinerten Sicht der gesellschaftlichen Gruppe als Ganzer, zu der er gehört" (Mead 1973: 180). Eingefangen wird mit dem Selbst auch die zeitliche Dimension, insofern es um dessen prospektive wie auch retrospektive Bearbeitung geht. Auf dieser dritten Ebene werden also Aspekte einer permanenten und umfassenden Selbstformung 
zusammengetragen - weit über eine rein berufsbezogene hinaus.

Zusammengeführt werden die drei Ebenen im Modell der Sozialisation als Selbstprojekt. Entwickelt wurde dieses Modell in der Auseinandersetzung mit den empirischen Daten. Im Folgenden werden entlang der Ebenen zentrale Ergebnisse der Studie präsentiert.

\section{EMPIRISCHE ANALYSE: SOZIALISATION ALS SELBSTPROJEKT IN BETRIEBEN UND HOCHSCHULEN}

Die Ergebnisse der Studie werden am Beispiel von vier ausgewählten Fällen präsentiert. Je zwei Fälle sind im Handwerk und im Studium verortet. Die ausgewählten Fälle erlauben, die unterschiedliche Strukturiertheit der Felder zu skizzieren (zu den Zugängen in die Felder: vgl. König 2018), eine feldspezifische Varianz in Hinblick auf Strategien wie auch Selbstformung im Zuge der Sozialisation aufzuzeigen. ${ }^{5}$

Jennifer und Marcus sind Auszubildende im Handwerk. Für beide ist der Ausbildungsstart holprig. Jennifer, ausgestattet mit einem Realschulabschluss, beginnt eine Ausbildung zur Bürokauffrau, die sie bald abbricht. Als nächstes fängt sie in einem Friseursalon an, in dem sie sich jedoch als „billige Arbeitskraft“ ausgenutzt fühlt. Im zweiten Interview ist der Wechsel in einen anderen Salon vollzogen. Mit dieser neuen Ausbildungsstätte ist sie rundum zufrieden. Dennoch, oder, wie wir sehen werden, deshalb, will sie das Feld des Handwerks nach Abschluss der Ausbildung verlassen. Marcus nimmt, nachdem er sein Ziel, einen Realschulabschluss zu erlangen, nicht erreicht hat, wenig begeistert die Lehre in einem Malerei-Lackier-Betrieb auf. Im ersten Interview hat er bereits einen Betriebswechsel vollzogen. ${ }^{6}$ Im vorherigen Betrieb war er nur für die „Drecksarbeit“ zuständig, im jetzigen ist „alles gut" und er erlernt das Handwerk. Dennoch ist er sich im zweiten Interview sicher, kein Maler-Lackierer zu sein bzw. zu werden. Das Feld des Handwerks will er verlassen und „im Büro“ arbeiten, wie die meisten Mitglieder seiner Familie. Den Weg dahin kennt er nicht.

Glenn und Caspar nehmen beide zögerlich ihr Studium auf, aus unterschiedlichen Gründen: Glenn, dessen Vater Ingenieur ist, zweifelt, ob er den Ansprüchen der Universität gewachsen sein wird. Er beginnt im Anschluss eine Ausbildung zum IT-Systemelektroniker ein Ingenieursstudium. Im zweiten Interview liebäugelt er gar mit einer Promotion. Für Caspar, dessen Vater ein Lehramtsstudium angefangen aber nicht abgeschlossen hatte, steht früh fest, dass er studieren wird. Nur, welcher Studiengang es sein soll, bleibt lange offen. Seine erste Wahl, ein kommunikationswissenschaftliches Studium, gibt er nach ein paar Monaten auf. Er fühlt sich überfordert - von der Mathematik aber auch der langen Pendelstrecke. Er wechselt ins Lehramt, womit sich für inn der Fahrweg verkürzt und die intellektuelle Herausforderung verkleinert. Ob er später Lehrer sein will, ist beim zweiten Interviewtermin nicht sicher.

\subsection{Sozialisation im Handwerksbetrieb}

Die Strukturiertheit des Feldes: Die Ausbildung zum/zur Maler-Lackiererln wie auch zum/zur Friseurln findet vor allem in kleinen Betrieben, in überschaubaren Arbeitsteams statt. Die Auszubildenden stehen dabei unter Beobachtung - von Vorgesetzten wie teilweise auch von Kundlnnen. Im Betrieb werden Positionen vor allem über das institutionalisierte Kapital bestimmt und hierarchisiert: Jennifer beschreibt, wie sie im ersten Salon „irgendwie dafür sorgen musste dass äh die wenigen Friseure die wir dann da hatten auch ihren Job machen konnten“. Eine kategoriale Differenz zwischen Friseurlnnen und Auszubildenden wird gezogen. Innerhalb der Gruppe der Auszubildenden werden Positionen nach Lehrjahren unterschieden. Gelegentlich gibt es Praktikantlnnen, die die niedrigste Position einnehmen. So erklärt es Marcus: „Praktikant und Azubi is normal dass der Praktikant dann immer nur die Drecksarbeit da macht". Das heißt, aus Sicht der Auszubildenden bestimmen sich die Positionen im Gefüge weniger über handwerkliches als über

\footnotetext{
${ }^{5}$ Das Datenmaterial der ausgewählten Fälle wurde bereits in der Monographie zur Studie präsentiert (König 2019). Im vorliegenden Aufsatz wird es zugespitzt aus einer sozialisationstheoretischen Perspektive gedeutet.

${ }^{6}$ Etwa jeder dritte Ausbildungsvertrag wird im Handwerk (NRW 2017) abgebrochen (BIBB-Datenblatt 99300020).
} 
institutionalisiertes Kapital. Mit den Positionen sind wiederum bestimmte Rechte und Pflichte verbunden. Allerdings hängen die zugeschriebenen Aufgaben nicht nur von der eigenen Position, sondern auch davon ab, ob niedrigere Positionen besetzt sind. Ist kein/e PraktikantIn angestellt, rücken keine neuen Auszubildenden nach, verändert sich die eigene Position weniger deutlich.

Das Spielgeschehen wird von zwei Interessen bestimmt, zum einem dem (geteilten) Interesse am Profit des Betriebs, und zum anderen dem Interesse der Auszubildenden, zur Handwerkerin bzw. zum Handwerker ausgebildet zu werden. Diese beiden Interessen können, müssen aber nicht, im Widerspruch zueinander stehen. Einige sind stolz auf ihren handwerklichen Beitrag für den Betrieb (Jennifer), andere klagen, nicht handwerklich arbeiten zu können, nicht „an den Kunden ran“ zu dürfen oder nicht "was mit Farbe zu tun" zu haben (Marcus zum ersten Betrieb). Die Abhängigkeit vom Meister und die eigene inferiore Position im Gefüge indiziert nicht zuletzt der häufig verwendete Ausspruch: „Lehrjahre sind ja sowieso keine Herrenjahre“ (Marcus). Es ist eine hierarchische und gleichzeitig familial geprägte Ordnung. So beklagt Jennifer, dass ihr erster Meister "sich nicht um die Auszubildenden gekümmert" hat. Ganz anders die KollegInnen im neuen Salon, die für sie, die seit ihrem 16. Lebensjahr alleine lebt, ein gewisser Familienersatz sind. Von ihnen erhält sie Zuspruch und Unterstützung. Der familiale Charakter kann aber auch mit persönlichen Angriffen und Beleidigungen einhergehen, wie sie aus dem privaten Raum bekannt sind. Marcus wie Jennifer wissen aus ihren ersten Betrieben davon zu berichten.

Das interaktive Geschehen und die Statusplatzierung im Feld: Sind die Positionen im Betrieb vor allem über das institutionalisierte Kapital bestimmt, so ist damit der Kampf um die Verbesserung der eigenen Position ein stückweit eingeschränkt. Dennoch sind Strategien zur Positionsbewahrung oder -verbesserung zu finden. So sind die Erzählungen gespickt mit Ordnungsrufen: Der Praktikant wird auf seinen Platz verwiesen, indem ihm der Besen in die Hand gedrückt wird; beschwert wird sich über die Auszubildende im ersten Lehrjahr, die sich nicht in die Ordnung fügt; der Meister wird daran erinnert, was man im dritten Lehrjahr laut
Ausbildungsrahmenplan können muss. Das heißt, die "theoretische Bestimmung“ des Ausbildungsrahmenplans wird in der "praktischen Wirklichkeit“" (Bourdieu 1999: 250) überwacht, gelegentlich aber auch uminterpretiert. So wird ab und an auf das eigene handwerkliche Kapital verwiesen, um - wider der Ordnung nach Lehrjahren - mehr Handlungsmöglichkeiten einzufordern.

Zwei zentrale Strategien fallen auf, die der Positionssicherung vor allem in problematischen Ausbildungsverhältnissen dienen: Eine zentrale Strategie derjenigen, die außerhalb des Betriebs wenig Chancen für sich sehen, gar den sozialen Abstieg fürchten, ist das Durchhalten. So hat Marcus die Mahnung seiner Mutter im Ohr: ,... auch wenn dir das nicht gefällt du musst auf jeden Fall durchziehen"; und von seinem Vater weiß er: „Da muss man durch halt“. Marcus teilt die Einschätzung seiner Eltern, denn „ohne Ausbildung ist man nichts in Deutschland“. Die eigenen Ansprüche an eine Ausbildung werden reduziert, um das Zertifikat zu erhalten. Durchhalten ist eine Strategie, die nicht bloß als „Erleiden" (Schütze 2006), denn auch als Kapital verstanden wird. Es ist eine Strategie derer, die aus einer inferioren Position heraus versuchen, den Verbleib im Spiel zu sichern; es ist eine Strategie vis-à-vis dem/der Meisterln. Legitimiert wird sie - etwa bei Marcus - durch den Verweis darauf, dass Lehrjahre keine Herrenjahre sind, was das Einfügen in die Ordnung als unausweichlich beschreibt. Es ist eine Komplizenschaft, mit der das hierarchische Gefüge gestützt und die weitere Teilnahme am Spiel gesichert wird. Eine andere Strategie, um die Position im Feld zu bewahren oder zu verbessern, ist die Akkumulation handwerklichen Kapitals außerhalb des Betriebs. Weil die Akkumulation handwerklichen Kapitals im Betrieb so stark vom Meister bzw. der Meisterin reglementiert (gefördert wie behindert) wird, ist der private Raum wichtig: Verwandte, die im gleichen Handwerk ausgebildet sind, vermitteln Kompetenzen; FreundInnen stellen ihre Köpfe zum Frisieren oder Wände zum Streichen zur Verfügung - solcherlei soziales Kapital wird in vielfältiger Weise genutzt.

Jennifer, die sich in ihrem zweiten Salon gut ausgebildet fühlt, ist auf außerbetriebliche Unterstützung nicht angewiesen. Sie akkumuliert 
handwerkliches Kapital innerhalb des Salons, bzw. wenn dies die Betriebslogik nicht erlaubt, ermöglicht ihr die Meisterin Übungsstunden in einer Akademie. Gleichzeitig sorgt Jennifer dafür, dass sie für den Salon profitabel ist. Wenn sie mal nicht gut drauf ist, putzt sie mit Ausdauer, denn „dann hab ich wenigstens was gemacht ne dann sitz ich nicht dumm da rum". Meisterin und Auszubildende kooperieren und handeln ihre Erwartungen wechselseitig aus.

Die Formung des Selbst: Das Eingewobensein in den organisierten „Erfahrungs- und Verhaltensprozeß" (Mead 1973: 301) des betrieblichen Geschehens ist grundlegend für die Vorbereitung auf das Berufsfeld Maler-Lackiererln bzw. Friseurln, mehr noch: für die Formung zur/zum Friseurln oder Malerln. Mit Jennifer und Marcus stehen zwei Auszubildende im Fokus, die nach Abschluss der Ausbildung nicht weiter in dem Beruf arbeiten werden. Unter denjenigen, die das Handwerk verlassen, konnten in der Gesamtstudie drei Typen identifiziert werden. Erstens sind da diejenigen, die mit dem Austritt die Modifizierung eines unpassenden Selbstprojekts vornehmen. Darunter fallen diejenigen, bei denen keine habituelle Passung vorliegt bzw. die mit der Wahl Gefahr laufen, ihren Status zu verspielen. Dazu zählen etwa AbiturientInnen oder Kinder aus Akademikerfamilien. Marcus steht für den zweiten Typus, der als Einschränkung des Selbstprojekts ohne Zukunftsentwurf bezeichnet werden kann. Er, der zu Beginn der Ausbildung froh war, irgendeine Ausbildung aufzunehmen, ist nach drei Jahren überzeugt, dass er kein Maler-Lackierer ist und sein will. Die Teilhabe an dem Spiel führt nicht zur Anpassung, sondern zur Abgrenzung. Die Orte der Arbeitseinsätze (.,wo die ganzen Assis sind“), die Kälte und Nässe auf dem Bau sind inm zuwider. Die Arbeit verspricht keinerlei symbolische Weihe. Er weiß, dass andere Auszubildende unter anderen Bedingungen arbeiten, aber eine Neu-Positionierung in dem Feld ist für ihn nicht mehr vorstellbar. Sein Wunschberuf wäre „[V]orm PC den ganzen Tag irgendwas da nen bisschen rumtippen schön im Warmen“. Wie ihm dieser Sprung gelingen soll, ist inm nicht klar. Hatte er vor Beginn der Ausbildung noch den Plan, seinen Realschulabschluss nachzuholen, sind seine Träume und Aspirationen nun zurechtgestutzt. Bourdieu spricht von der "Trauerarbeit“ der Beherrschten, „ihre Wünsche und Erwartungen den jeweils objektiven Chancen anzugleichen und sich in ihre Lage zu fügen" (1999: 189). Die Durchhalteparolen der Eltern befördern dies. Spürbar wird bei Marcus auch der starke Zeitdruck - typisch für Jugendliche aus weniger privilegierten Familien -, der eine Revision von Entscheidungen kaum zulässt (Reißig 2015). So reagiert Marcus auf die (ungeschickte) Frage der Interviewerin, ob er eventuell eine andere Ausbildung anschließen wird, irritiert. Dies ist nicht denkbar, „weil wenn ich jetzt erst nach der Ausbildung mal Realschulabschluss machen würde ein Jahr oder nochmal drei Jahre Industriekaufmann. Bin ich ja fast in Rente."

Die Analyse der Zukunftsentwürfe der Auszubildenden zeigt, dass die Trauerarbeit nicht unweigerlich mit einem Wissen darüber einhergeht, wie der passende Weg nun aussehen könnte eine „Fügung" entsprechend "seiner Lage“, wie es mit Bourdieu zu erwarten wäre, ist (noch) nicht erkennbar.

Neben der Trauerarbeit kann auch eine Erweiterungsarbeit identifiziert werden. Hier wird der Möglichkeitsraum für das Selbstprojekt durch die Tätigkeit im Betrieb (ergänzt um Zwischenrufe vom Spielfeldrand) erweitert. Für dieses dritte Muster, das als Erweiterung des Möglichkeitsraums für das Selbstprojekt verstanden werden kann, steht Jennifer. Die rundum positive Bewertung der Ausbildung, das freundschaftliche Verhältnis zu den Kolleglnnen, die Anerkennung ihrer handwerklichen Leistung, der Spaß an der Arbeit - all dies führt zu einer Selbstformung als Friseurin. Jennifer ist stolz über ihre Entwicklung, will an diesem Punkt aber nicht stehenbleiben. Durch die Ausbildung hat sie, die es im Leben nicht immer leicht hatte, die Erfahrung gemacht, alles schaffen zu können. Genau damit wächst ihre Status- wie auch Selbstorientierung: Abitur, Studium, eine andere Ausbildung - alles scheint nun möglich. Unterstützt wird diese Selbst-Erweiterung durch Reaktionen feldexterner Personen, die sie wiederholt mit der Frage konfrontieren: „Wieso bist du Friseurin? Du kannst doch voll viele andere Sachen machen". Sie sieht sich durch die Augen der anderen, die keine Selbst-Passung sehen und überlegt, „ob ich das wirklich mein ganzes Leben lang machen will". Zum Zeitpunkt des zweiten Interviews ist 
ihre Antwort eindeutig nein. Die offene Zukunft empfindet sie nun - ganz anders als zu t1 - als große Freiheit.

\subsection{Sozialisation an der Hochschule}

Die Strukturiertheit des Feldes: Das Lehramtswie auch das ingenieurswissenschaftliche Studium findet vor allem in zahlenmäßig starken Studiengängen statt. Kennzeichnend für die Großveranstaltungen ist, dass die Anwesenheit der Studierenden für die Dozierenden nicht unmittelbar relevant ist, teils gar nicht verlangt wird. Die Dozierenden - in kategorialer Differenz zu den Studierenden - treten in den ersten Interviews vor allem als Personen auf, die Punkte vergeben und die Studienordnung repräsentieren. Das unpersönliche Verhältnis geht mit dem Eindruck einher, dass alle unter gleichen Bedingungen studieren. Beschrieben wird eine universalistische Ordnung mit teils schulähnlichem Charakter. Wann was gelernt werden soll, ist vorgegeben. Darüber wachen, dass sie lernen, was sie laut Studienordnung lernen sollen, müssen die Studierenden nicht. Klagen bestehen hingegen in Bezug auf die Begrenztheit der Wahlmöglichkeiten. Die Freiheit besteht, so Caspar, vor allem darin, zu entscheiden, wie „viel man machen will und ob man eventuell also ob man morgens zur Uni will oder halt nicht." Verlangt ist ein hohes Maß an Selbstmanagement genau das unterscheidet die Universität für viele Befragte von der Schule. Und es irritiert - in Kombination mit dem unpersönlichen generationalen Arrangement - insbesondere jene, die eine unwahrscheinliche Wahl getroffen haben (Schmitt 2010). Die Leerstelle füllen vor allem die KommilitonInnen. In Konkurrenz stehen die Studierenden dabei kaum untereinander. Ihr gemeinsames Interesse besteht vor allem darin, das studentische Leben zu zelebrieren, sich bei der Organisation des Studiums zu unterstützen bzw. - vor allem unter den ingenieurswissenschaftlichen Studierenden - gemeinsam die Prüfungsanforderungen zu meistern. Letzteres wird durch die vorgegebene Studienstruktur gestützt, die das gemeinsame Besuchen von Veranstaltungen (ähnlich dem Klassenverband) ermöglicht. Entsprechend ist das Bestehen von Prüfungen wichtig, um in der Arbeits- und Lerngruppe zu bleiben. Die einzelnen Noten sind jedoch kaum Kapital innerhalb des Feldes. Glenn ist einer der wenigen, der Profit aus seinen sehr guten Leistungen schlagen kann: Er bekommt eine Stelle als studentische Hilfskraft angeboten. In dieser universitätsinternen Subwelt bekommt er exklusive Einblicke in Forschungsarbeiten, arbeitet mit Doktorandlnnen zusammen, erhält Feedback für seine Arbeit und Informationen über das ingenieurswissenschaftliche Feld. Hier erspürt er die wissenschaftliche illusio - anders als im Studium, bei dem er das Bestehen der Prüfungen als das zentrale Interesse wahrnimmt.

Das interaktive Geschehen und die Statusplatzierung im Feld: Im Laufe des Studiums erfahren die Studierenden, welche Investitionen notwendig sind, um im Spiel zu bleiben, also die Prüfungsleistungen zu erfüllen. Hinweise darüber erhalten sie von erfahreneren KommilitonInnen sowie über die ersten Prüfungen, die sie (nicht) meistern. An die so eruierten Anforderungen, die detaillierter sind als jedes Modulhandbuch, passen sie ihre Investitionen an. Für Medizinstudierende haben bereits Becker et al. (1961) eindrücklich gezeigt, wie diese die Erwartungen des Lehrkörpers zu erspüren suchen und sich daran orientieren. Das heißt, die Strategien, die wir bei den heutigen Studierenden sehen, sind mitnichten neu. Allerdings gibt es (studiengangs)spezifische Herausforderungen. So folgt die Anpassung der damaligen Medizinstudierenden der Einsicht, dass sie die Menge des Studienstoffs nicht verarbeiten können und Reduktionsstrategien unvermeidbar sind. Hingegen erzählen vor allem Lehramts- aber auch Ingenieursstudierende, wie sie mit weniger Aufwand durch das Studium gelangen. Beachtlich ist das differentielle Wissen, das sie im Laufe des Studiums (nicht unähnlich den „Boys in White") entwickeln. Die Strategie der Reduktion der Investition lässt sich vor allem bei jenen nachzeichnen, die überraschend Klausuren bestehen. Caspar beispielsweise ist zu Beginn der Aufnahme des zweiten Studiengangs etwas nervös, ob er hier ähnliche Schwierigkeiten haben wird, wie im ersten Studiengang. Die Sorge legt sich „relativ schnell eigentlich [...] als ich dann die ersten Klausuren geschrieben hab und so und auch gemerkt hab das geht eigentlich alles". Seine weitere Strategie erklärt er mit Blick auf die Studienordnung: Sein Engagement will er ganz auf die Abschlussprüfungen konzentrieren, die mit großem Gewicht in die Gesamtbeurteilung 
Eingang finden. Bis dahin minimiert er seine Investitionen - zugunsten von Arbeit und Freizeit.

Es gibt auch Studierende, die der Strategie der Erhöhung der Investitionen folgen. Glenn ist ein Beispiel. Er erzählt nicht ohne Stolz, dass er auch in den Klausuren mit den höchsten Durchfallquoten "locker durchgekommen“" ist. Glenns Sorge, dem universitären Anspruch nicht gewachsen zu sein, war zu Beginn ausgeprägter als bei Caspar. Er reduziert seine Investitionen nach solchen positiven Klausurerfahrungen jedoch nicht. Was ist bei ihm anders? Am „Anfang dacht ich mir echt dann joa...könnte... Hauptsache irgendwie schaffen [...]. Aber mittlerweile hat man dann vielleicht doch andere Ansprüche wenn man weiß was möglich ist". Den Möglichkeitsraum, den er für sich erkennt, will er nun nutzen. Nicht nur seine Vorerfahrung in der Ausbildung motiviert inn, sondern insbesondere die Arbeit am Lehrstuhl, wo er die wissenschaftliche illusio kennenlernt und einverleibt.

Mit Blick auf die Studierenden ist als eine weitere Strategie die des Durchziehens interessant - gerade in Abgrenzung zu der des "Durchhaltens", wie sie im Handwerk zu finden ist. Caspar ist ein Vertreter. „Also...für mich ist eigentlich auch für mich persönlich wichtig dass ich einfach das Studium erstmal durchziehe...einfach so dass ich also.... weil ich das das also ungern jetzt irgendwas so abbreche." Das Durchziehen ist keine Strategie vis-à-vis einer vorgesetzten Person (wie das „Durchhalten“ der Auszubildenden gegenüber dem Meister) noch ist es von Abstiegsängsten durchtränkt. Zwar geht es auch hier um das Erreichen des Abschlusses, allerdings stärker im Sinne eines Selbst-Anspruchs.

Die Formung des Selbst: Zum Zeitpunkt des zweiten Interviews stehen die Studierenden in der Regel kurz vor Abschluss des BA-Studiums. ${ }^{7}$ Der Anschluss eines Master-Studiums steht für die meisten - so auch für Glenn und Caspar fest. Das Studium hat bis dahin weniger die Transformation zum/zur Lehrerln oder zum/zur Ingenieurln angestoßen als die Transformation zum Studenten bzw. zur Studentin. Eine umfassende Transformation ist auch dies nicht. So wird zum einen aus biographischer Perspektive kein wesentlicher Bruch zur schulischen Lebensweise wahrgenommen. Zum anderen ist, mit Blick auf die Gegenwart, das Studium nicht die einzig prägende Lebenswelt - sie steht neben Arbeit und Freizeit (Stichweh 2016). Demgemäß absorbiert das Studium nicht so viel Energie wie wir es bei den Auszubildenden sehen. Reduzieren letztere abendliche Unternehmungen, so gehören die Treffen und Feiern mit KommilitonInnen außerhalb der Klausurphasen zum Inbegriff des studentischen Lebens. Auffallend ist auch, dass das erworbene fachliche Wissen kaum relevantes Thema ist - weder außerhalb der Universität noch im Interview. Drei Muster der Bezugnahme zu fachlichen Inhalten können in der Gesamtstudie aus den Interviews herausgearbeitet werden: 1) ein funktionaler Bezug, der orientiert ist an dem späteren beruflichen Selbst (vor allem bei den Lehramtsstudierenden), 2) ein hoher Selbstbezug zum Fach und 3) ein loser Bezug zum Fach. Für das zweite Muster steht Glenn, der insbesondere durch das Eingewobensein in die Arbeit am Lehrstuhl eine Fachidentität entwickelt. Zunehmend teilt er die illusio des wissenschaftlichen Feldes und versucht sich in diesem zu positionieren. Das dritte Muster verkörpert Caspar, für den Studium und Beruf nur lose miteinander verbunden sind. Dennoch, er genießt das Studium bzw. das studentische Leben als ein Moratorium, das inn von vielen Zwängen und Verpflichtungen in akzeptierter Weise freistellt. Gerade unter den Lehramtsstudierenden ist eine gewisse Pfadabhängigkeit der nachuniversitären Schritte erkennbar. Caspar hat beispielsweise mit dem Studium eine zu ihm passende Wahl getroffen, dessen Fortsetzung für ihn außer Frage steht. Ob die Studienfachwahl die beste ist, weiß er nicht. Aber mit der Fortführung des Studiums kommt er der Schule automatisch näher, d.h. „es wird wahrscheinlich so sein, dass ich natürlich das Referendariat mache, weil das ist ja einfach das Logischste, was sich dann auch ergibt“. Für beide Studierendengruppen ist zu t2 charakteristisch, dass sie sich ausbildungsadäquat entwerfen. Dass sie in den anvisierten Berufen einsteigen

\footnotetext{
${ }^{7}$ Eine Befragung von Absolventlnnen des Jahrgangs 2012 zeigt, dass die Abbruchquote unter Ingenieurswissenschaften mit 36\% vergleichsweise hoch ist (Heublein et al. 2014: 3 f.). Unter denjenigen, die zwischen 2006 und 2008 ihr Lehramtsstudium aufgenommen haben, liegt die Abbruchquote bei 12\%.
} 
können, wird kaum infrage gestellt - das institutionalisierte Kapital wird den Zugang sicherstellen.

\section{FAZIT}

Das Modell der "Sozialisation als Selbstprojekt" ist ein Vorschlag, um Sozialisation gleichermaBen aus interaktionistischer und ungleichheitsanalytischer Perspektive zu erfassen. Der vorliegende Beitrag stellt das Modell anhand einer qualitativen Längsschnittstudie vor, in der es ausgearbeitet wurde. Diese Studie wendet sich der Ausbildung in kleinen Handwerksbetrieben sowie dem Lehramts- und Ingenieursstudium an Hochschulen zu und fasst diese als Statuspassagen, die auf die Vorbereitung beruflicher bzw. gesellschaftlicher Teilhabe ausgerichtet sind. Bereits Durkheim hat darauf aufmerksam gemacht, dass Sozialisation immer in Hinblick auf eine bestimmte soziale Ordnung bzw. Position geschieht. Entsprechend interessiert hier, wie Sozialisation in Betrieben und Hochschulen verläuft und auf welche Ordnungen und Positionen die jungen Erwachsenen vorbereitet werden. Grundannahme ist, dass nicht erst das Zertifikat Positionen eröffnet oder verschließt, sondern bereits im und durch das Studium bzw. die Ausbildung ungleiche Chancen hergestellt werden. Wie Ungleichheit durch die Statuspassage produziert wird, ist die erste zentrale Frage der Studie. Zweitens wird die Statuspassage nicht bloß als Vorbereitung auf die soziale Position im späteren Erwachsenenalter verstanden. Vielmehr wird die Statuspassage mit Blick auf die Gegenwart der jungen Erwachsenen betrachtet. Herausgearbeitet werden kann durch die gemeinsame Betrachtung von Studium und Ausbildung dann die Ungleichheit der Statuspassagen.

Abschließend werden nun nicht die Analyseergebnisse systematisch vergleichend zusammengefasst. Vielmehr wird der heuristische Wert des vorgeschlagenen Modells diskutiert und an ausgewählten Befunden aufgezeigt. Schlüsselkonzept des Modells ist das Selbstprojekt, das drei Ebenen bündelt:

Auf einer ersten Ebene wird Sozialisation innerhalb eines spezifisch strukturierten Feldes verortet. Mit Blick auf die Auszubildenden und die
Studierenden zeigt sich, wie unterschiedlich die Statuspassage strukturiert sein kann: Während sich die Studierenden in einer schulähnlichen, universalistischen Ordnung bewegen, die auf der Idee der Meritokratie basiert, finden sich die Auszubildenden in einem hierarchisch-familialen Gefüge platziert, das durch die illusio geprägt ist, dass das Handwerk "goldenen Boden“ habe und von allen erlernt werden könne. Entsprechend bestehen je nach Feld andere Barrieren für die Spielteilnahme: Die Hochschule betreten vor allem jene zögerlich, die eine unwahrscheinliche Wahl getroffen haben, in dem Sinne, dass sie aus einem eher bildungsfernen Elternhaus stammen - dies verwundert aus bourdieuscher Perspektive kaum. Zögerliche Spielaufnahmen gehen hier mit dem Zweifel an der eigenen Befähigung einher. In den Ausbildungsbetrieben sind es hingegen nicht die unwahrscheinlichen Wahlen - in Bezug auf soziale Herkunft und Abitur -, die mit einem Stocken einhergehen. Vielmehr ist die Spielteilnahme beeinträchtigt, sobald sich die Auszubildenden auf Hilfstätigkeiten begrenzt und damit in der beruflichen Selbstformung eingeschränkt fühlen - die illusio des Feldes wird so brüchig. Das Feldkonzept ist nicht eins-zu-eins auf den gewählten Untersuchungsgegenstand anwendbar - so ist beispielsweise der Kampf um Positionen in Ausbildungsbetrieben eingeschränkt. Gleichwohl gestattet das adaptierte Feldkonzept, die Strukturiertheit der Betriebe und Hochschulen einzufangen und die Positionen der jungen Erwachsenen in dem relationalen Gefüge zu bestimmen. Eingenommen wird so eine ungleichheitsanalytische Perspektive, die es erlaubt, die Ungleichheiten der Statuspassagen zu erfassen und die Strategien der AkteurInnen zu positionieren und zu kontextualisieren.

Auf einer zweiten Ebene wird Sozialisation als interaktives Geschehen gefasst. Auf dieser Ebene der Heuristik setzt sich die interaktionistische Perspektive durch. So wird die Frage nach der habituellen Vorangepasstheit geöffnet zur Frage nach der interaktiven Anpassung. Die gemeinsame Betrachtung von Studierenden und Auszubildenden zeigt, dass die beiden Gruppen vor ganz anderen Herausforderungen stehen. Ein Beispiel ist die Möglichkeit des Erwerbs feldspezifischen Kapitals: Einige Auszubildende Klagen über die durch den/die Meisterln eingeschränkten Gelegenheiten, Frisieren oder 
Malen zu lernen. Eine Lösung ist es, von Familienmitgliedern Tipps zu erhalten, die im gleichen Handwerk tätig sind, um zumindest die Prüfungen bestehen zu können. Für Studierende ist die Möglichkeit, fachliche Inhalte zu erlernen, nicht in ähnlicher Weise durch das relationale Gefüge reglementiert. Insgesamt spricht die Studie auf theoretischer Ebene dafür, die Bindung zwischen Strategien und sozialer Herkunft offener zu fassen als eine orthodoxe Bourdieu-Auslegung nahelegen würde, eben im Sinne einer interaktiven Anpassung. Dazu zählt dann auch, dass als Interaktionspartnerlnnen nicht nur Feldteilnehmerlnnen, sondern auch signifikante Andere relevant sind, die nicht unmittelbar am Feldgeschehen teilnehmen. Mehr noch, die Analyse zeigt, dass die Felder unterschiedlich anfällig sind für solche feldexternen Interventionen: So schlagen sich etwa im Studium Ereignisse im privaten Raum nicht so unmittelbar im Geschehen nieder wie im Ausbildungsbetrieb, in den private Probleme direkter hineingetragen werden oder zumindest wahrnehmbarer sind als in anonymeren Studiengängen mit großen Kohorten.

Auf einer dritten Ebene wird Sozialisation als Prozess der Formung des Selbst verstanden. Das Verwickeltsein in eine Handlungslinie bedeutet eben nicht nur etwas zu tun, sondern auch, etwas zu sein bzw. zu werden - so Strauss (1974: 40) in Anlehnung an Burke. Dabei geht die Selbstformierung weit über eine rein berufliche hinaus und ist auch durch das Interaktionsgeflecht mit feldexternen Akteurlnnen geprägt. Kennzeichnend für die Auszubildenden ist beispielsweise, dass ihre Fähigkeiten auch im privaten Raum nachgefragt werden: Freundlnnen werden frisiert, die Wohnung der Eltern renoviert. Dies trägt nicht nur zur beruflichen Selbstformierung bei, sondern verändert auch ergänzt teilweise um Einzahlungen in die Familienkasse - die Position im familialen Gefüge. Bei den untersuchten Studierendengruppen hingegen werden kaum Kompetenzen im privaten Raum nachgefragt. Eine neue Selbstständigkeit erfahren sie vor allem durch einen Auszug, womit sich bei innen das familiale Gefüge etwas verschiebt. Mit dem Selbst-Konzept ist die Prozesshaftigkeit und das "working out" besser zu erfassen als mit dem Habituskonzept. Deutlich wird dies am Material etwa in Bezug auf die "Trauerarbeit", womit Bourdieu das Aufgeben von „unpassenden“ Zielen und zu hohen Statusaspirationen der Beherrschten bezeichnet. Die vorliegende Studie ergänzt, dass „Trauerarbeit“ vor allem in den stark asymmetrischen Gefügen von Betrieben oder/und in Betrieben stattfindet, die wenig symbolische Weihung versprechen. Das heißt, nicht allein die soziale Herkunft erklärt die "Trauerarbeit" der Beherrschten, vielmehr wird soziale Ungleichheit in und durch die jeweilige Logik und Struktur der Felder in spezifischer Weise hergestellt.

Im Modell der "Sozialisation als Selbstprojekt" werden diese drei Ebenen zusammengeführt, mit dem Anspruch, den jeweiligen analytischen Zuspitzungen interaktionistischer wie ungleichheitsanalytischer Perspektiven entgegenzuwirken. Erinnert sei an das in der Analyse entstandene Konzept der "Erweiterungsarbeit" als Komplementär zur „Trauerarbeit". Für die Forschung erlaubt das Modell, Studierende und Auszubildende unter einer gemeinsamen sozialisationstheoretischen Perspektive zu betrachten und so Ungleichheiten der Statuspassage sowie Ungleichheiten, die durch die (Feldspezifik der) Statuspassage evoziert wird, zu untersuchen

\section{LITERATUR}

Abels, Heinz/König, Alexandra (2016): Sozialisation. Über die Vermittlung von Gesellschaft und Individuum und die Bedingungen von Identität. 2. Aufl. Wiesbaden: VS. https://doi.org/10.1007/978-3-658-13229-3

Becker, Howard S./Geer, Blanche/Hughes, Everett C./Strauss, Anselm L. (1961): Boys in White. Student Culture in Medical School. Chicago: University of Chicago Press.

Becker, Rolf (2009). Entstehung und Reproduktion dauerhafter Bildungsungleichheiten. In: Becker, Rolf (Hrsg.): Lehrbuch der Bildungssoziologie. Wiesbaden: VS, S. 85-129. https://doi.org/10.1007/978-3-531-91711-5_4

BIBB-Datenblatt 99300020: Handwerk. NRW. https://www.bibb.de/tools/dazubi/data/Z/B/5/99300020.pdf (09.11.2019).

Bourdieu, Pierre (1999): Die feinen Unterschiede. Kritik der gesellschaftlichen Urteilskraft. Frankfurt am Main: Suhrkamp. 
Bourdieu, Pierre (2001a): Meditationen. Zur Kritik der scholastischen Vernunft. Frankfurt am Main: Suhrkamp.

Bourdieu, Pierre (2001b): Habitus, Herrschaft und Freiheit. Interview mit Antoine Spire, Pascale Casanova und Miguel Banassayag. In: Bourdieu, Pierre: Wie die Kultur zum Bauern kommt. Über Bildung, Schule und Politik. Hamburg: VSA, S. 162-173.

Bourdieu, Pierre (Hrsg.) (2002): Der Einzige und sein Eigenheim. Hamburg: VSA.

Bourdieu, Pierre/Passeron, Jean-Claude (1971): Die Illusion der Chancengleichheit. Stuttgart: Klett.

Bourdieu, Pierre/Wacquant, Loïc D. J. (1996): Die Ziele der reflexiven Soziologie. In: Bourdieu, Pierre/Wacquant, Loïc D. J. (Hrsg.): Reflexive Anthropologie. Chicago-Seminar Winter 1987. Frankfurt am Main: Suhrkamp, S. 95-249.

Bühler-Niederberger, Doris (2019): Lebensphase Kindheit. Theoretische Ansätze, Akteure und Handlungsräume. 2. Aufl. Weinheim: Beltz.

Bühler-Niederberger, Doris/Türkyilmaz, Aytüre (2014): Sozialisation als generationales Ordnen ein theoretischer und empirischer Versuch. In: Zeitschrift für Soziologie der Erziehung und Sozialisation 34 (4), S. 339-354.

Emmerich, Marcus (2016): Organisierte Erziehung und kategoriale Ungleichheit. In: Leemann, Regula Julia/Imdorf, Christian/Powell, Justin J. W./Sertl, Michael (Hrsg.): Die Organisation von Bildung. Soziologische Analysen zu Schule, Berufsbildung, Hochschule und Weiterbildung. Weinheim/Basel: Beltz Juventa, S. 126-146.

Engler, Steffani (1993): Fachkultur, Geschlecht und soziale Reproduktion. Eine Untersuchung über Studentinnen und Studenten der Erziehungswissenschaft, Rechtswissenschaft, Elektrotechnik und des Maschinenbaus. Weinheim: Deutscher Studien Verlag.

Friebertshäuser, Barbara (2008): Statuspasssage von der Schule ins Studium. In: Helsper, Werner/Böhme, Jeanette (Hrsg.): Handbuch der Schulforschung. 2. Aufl. Wiesbaden: VS, S. 611627. https://doi.org/10.1007/978-3-53191095-6_24
Georg, Werner/Sauer, Carsten/Wöhler, Thomas (2009): Studentische Fachkulturen und Lebensstilen - Reproduktion oder Sozialisation? In: Kriwy, Peter (Hrsg.): Klein aber fein! Quantitative empirische Sozialforschung mit kleinen Fallzahlen. Wiesbaden: VS, S. 351-372. https://doi.org/10.1007/978-3-531-913803_14

Glaser, Barney G./Strauss, Anselm L. (2010): Status Passage. New Brunswick/London: Aldine Transaction.

Heinz, Walter R./Kelle, Udo/Witzel, Andreas/Zinn, Jens (1998): Vocational Training and Career Development in Germany: Results from a Longitudinal Study. In: International Journal of Behavioral Development 22 (1), S. 77-101. https://doi.org/10.1080/016502598384522

Heublein, Ulrich/Richter, Johanna/Schmelzer, Robert/Sommer, Dieter (2014): Die Entwicklung der Studienabbruchquoten an den deutschen Hochschulen Statistische Berechnungen auf der Basis des Absolventenjahrgangs 2012. Hannover: DZHW.

Hillmert, Steffen (2014): Bildung, Ausbildung und soziale Ungleichheiten im Lebenslauf. In: Zeitschrift für Erziehungswissenschaft 17 (2), S. 73-94. https://doi.org/10.1007/s11618-0130465-2

Isleib, Sören (2019): Soziale Herkunft und Studienabbruch im Bachelor- und Masterstudium. In: Lörz, Markus/Quast, Heiko (Hrsg.): Bildungs- und Berufsverläufe mit Bachelor und Master. Determinanten, Herausforderungen und Konsequenzen. Wiesbaden: VS, S. 307-337. https://doi.org/10.1007/978-3-658-22394$\underline{6 \_10}$

Klaus, Sebastian (2012): Biographie und vorzeitige Vertragslösung. Erkenntnisse über die Entwicklungsgeschichte der Betroffenen. In: BIOS Zeitschrift für Biographieforschung, Oral History und Lebensverlaufsanalysen 25 (2), S. 204-227. https://doi.org/10.3224/bios.v31

König, Alexandra (2018): Von wahrscheinlichen und unwahrscheinlichen Wegen: Der Zugang zum Friseursalon, zur Universität und zur Kunstakademie im kontrastiven Vergleich. In: Zeitschrift für Soziologie der Erziehung und Sozialisation 38 (3), S. 250-266. 
König, Alexandra (2019): Spielfelder des Selbst. Eine Längsschnittstudie zu jungen Erwachsenen in Handwerksbetrieben, Hochschulen und Kunstakademien. Weinheim/Basel: Beltz Juventa.

Kreher, Thomas (2007): ,Heutzutage muss man kämpfen: Bewältigungsformen junger Männer angesichts entgrenzter Übergänge in Arbeit. Weinheim/München: Juventa.

Lehmann, Wolfgang (2009): Becoming Middle Class: How Working-Class University Students Draw and Transgress Moral Class Boundaries. In: Soziologie 43 (4), S. 631-647. https://doi.org/10.1177/0038038509105412

Mead, George Herbert (1973): Geist, Identität und Gesellschaft. Frankfurt am Main: Suhrkamp.

Niederbacher, Arne/Herbertz, Oliver/Hitzler, Ronald (2010): Studierende: Die unbekannten Wesen. In: Technische Universität Dortmund (Hrsg.): Mittendrin: Wie es sich anfühlt, Student zu sein. Bönen: Kettler, S. 21-23.

Reay, Diane/Crozier, Gill/Clayton, John (2009): ,Strangers in Paradise?: Working-Class Students in Elite Universities. In: Sociology 43 (6), S. 1103-1121.

\section{https://doi.org/10.1177/0038038509345700}

Reißig, Birgit (2015): Übergänge von der Schule in den Beruf. In: Sandring, Sabine/Helsper,
Werner/Krüger, Heinz-Hermann (Hrsg.): Jugend. Theoriediskurse und Forschungsfelder, Bd. 2. Wiesbaden: VS, S. 187-207. https://doi.org/10.1007/978-3-658-03543-3

Schmitt, Lars (2010): Bestellt und nicht abgeholt. Soziale Ungleichheit und Habitus-Struktur-Konflikte. Wiesbaden: VS. https://doi.org/10.1007/978-3-531-92193-8_5

Stichweh, Rudolf (2016): Studentische Lebensführung. In: Erika Alleweldt, Erika/Röcke, Anja Röcke/Steinbicker, Jochen (Hrsg.): Lebensführung heute. Klasse, Bildung, Individualität. Weinheim/Basel: Beltz Juventa, S. 140-147.

Strauss, Anselm L. (1974): Spiegel und Masken. Die Suche nach Identität. Frankfurt am Main: Suhrkamp.

Strauss, Anselm L. (1991): Grundlagen qualitativer Sozialforschung. München: UTB.

Strauss, Anselm L. (2014): Continual Permutations of Action. New Brunswick/London: Aldine Transaction.

Witzel, Andreas (2000): The Problem-Centered Interview. In: Forum Qualitative Sozialforschung/Forum: Qualitative Social Research 1 (1) http://www.qualitative-research.net/index.php/fqs/article/viewFile/1132/2520 (05.11.2019).

\section{Zur Autorin}

Alexandra König ist Professorin für Sozialisationsforschung an der Fakultät für Bildungswissenschaften an der Universität Duisburg-Essen. Sie ist Mitglied im Vorstand der DGS-Sektion Bildung und Erziehung und forscht zu Sozialisation und Bildung, Kindheit und Jugend, Kultur und Ästhetik.

Kontakt

Prof. Dr. Alexandra König

Universität Duisburg-Essen

Fakultät für Bildungswissenschaften

AG Sozialisationsforschung

Universitätsstr. 2

45141 Essen

Tel.: +492011836022

E-Mail: alexandra.koenig@uni-due.de

URL: https://www.uni-due.de/biwi/koenig/koenig.php 